\title{
Tribology properties of hybrid graphene oxide materials as lubricant additives
}

\author{
Anastasia A. Novikova*, Victoria E. Burlakova, Valery N. Varavka, \\ Tatyana G. Statsenko, Grigory B. Kharitonov and Vladilen V. Rubanov \\ Don State Technical University, 344000 Rostov-on-Don pl. Gagarina 1, Russia
}

\begin{abstract}
Graphene oxide was synthesized by the modified Hammers method. With managed hydrolysis in isopropanol solution obtained hybrid material "graphene oxide - copper oxide nanoparticles". The phase composition of the hybrid material was studied by X-ray phase analysis and UV-visible spectroscopy. By ultrasonic processing dispersions of synthesized materials in glycerol were produced. The concentration of lubricating additives in the lube oil was 0.05 wt. \%. The tribological properties of dispersions were investigated using a pin-on-disc friction machine. Tests showed that in the presence of graphene oxide, the friction coefficient was $\sim 0.02$, while with the addition of a hybrid material, the coefficient of friction was $\sim 0.035$. This is due to various mechanisms of lubrication. Reduction of the coefficient of friction in the presence of graphene oxide is associated with the formation of tribocarbon on the porosity of frictional contacts. While the addition of a hybrid material containing the $\mathrm{CuO}$ nanoparticles leads to the formation of a third body.
\end{abstract}

\section{Introduction}

To modern lubricants, in addition to the requirements of efficiency and economy, reducing the frictional load, there are strict requirements for environmental cleanliness and safety. In this regard, the search for a new class of compounds that could become the basis or part of the lubrication system seems to be an extremely topical direction. The development of methods for the synthesis and study of the properties of graphene is one of the most actively developing areas of materials science, physics and chemistry. Graphene is a single plane, wherein $\mathrm{sp}^{2}$-hybridized carbon atoms form two-dimensional hexagonal lattice [1, 2]. The graphene attracts attention to application in tribology [3, 4]. Graphene possess a number of unique properties: a high Young's modulus and corrosion resistance, an extremely high thermal and electrical conductivity [5-7]. Graphene can be used not only as a solid lubricant, but also as an additive to conventional lubricants, water and other types of solvents [8].

One of the most suitable materials for green tribology is graphene oxide [9-10]. Study tribological features of colloidal graphene oxide dispersions in various solvents is a promising direction. The study of the mechanism of lubricating action of graphene

\footnotetext{
*Corresponding author: anastasianovik@mail.ru
} 
materials is the subject of active research $[11,12]$. It is assumed that the decrease in the friction coefficient is achieved due to the formation of a tribofilm preventing metal-to-metal contact and providing a slip between the graphene layers based on a weak Van-der-Waals interaction [13]. The stability of this film depends strongly on the test conditions, the composition and morphology of graphene materials [14].

The authors of [15] investigated the lubricating effect of graphene dispersions in ethanol in a steel-steel friction pair. It was established that strength and stability of graphene tribofilm is low, after the evaporation of the solvent, an increase in the coefficient of friction occurs. The non-stability of the tribofilm under conditions of high loads limits the possibility of using unmodified graphene in the composition of lubricants. The solution to this problem is the creation of hybrid composite materials based on graphene. To date, tribotechnical hybrid graphene materials have been synthesized: $\mathrm{BN}_{-} \mathrm{TiO}_{2}$-graphene oxide [16], AlOOH-graphene oxide [17], $\mathrm{MoSe}_{2}$-reduced graphene oxide [18], $\alpha-\mathrm{Fe}_{2} \mathrm{O}_{3}$-reduced graphene oxide [19]. Thus, the synthesis and study of tribotechnical capabilities of graphene materials is an urgent scientific task.

\section{Materials and methods of research}

\subsection{Preparation of Graphene Oxide and Hybrid "GO-CuO"}

Graphite oxide was obtained by the modified Hammers method. In $100 \mathrm{ml}$ of $96 \%$ sulfuric acid, a finely divided graphite powder and sodium nitrate were mixed. Components were thoroughly mixed in a desiccator under external ice cooling. At the same time, potassium permanganate was gradually introduced into the mixture. The reaction mixture was thickened for 30 minutes, after which distilled water was slowly added to the resulting paste. After 30 minutes, an excess of distilled water and hydrogen peroxide were added. After that, the precipitate of graphite oxide was separated and dried. The graphite oxide suspensions in glycerol are dispersed in an ultrasonic bath for 45 minutes to produce graphene oxide $(\mathrm{GO})$.

The GO was sonicated for 45 minutes in isopropanol. The $\mathrm{GO}$ dispersion and $\mathrm{CuCl}_{2}$ were placed in a three-necked flask with condenser, thermometer and stirrer. The mixture was kept at $83{ }^{\circ} \mathrm{C}$ for 30 minutes. Then distilled water, $\mathrm{KOH}$ and $\mathrm{NH}_{4} \mathrm{OH}$ were rapidly added and heating was continued for 30 minutes. The obtained black precipitate was washed distilled water and centrifuged.

\subsection{Characterization Techniques}

The GO and GO-CuO were characterized by means of powder X-ray diffraction (XRD, ARL 'Xtra, filtered $\mathrm{Cu} \mathrm{K} \mathrm{K}_{\alpha}$ radiation, $\lambda=1,5406 \AA$ ). The pattern was recorded in the $2 \theta$ range between $2^{\circ}$ and $60^{\circ}$ (step size $0.02^{\circ}$, step time $1 \mathrm{~s}$ ). Inter-planar distances were calculated using the Bragg equation. The UV-Vis spectra of GO in glycerol solution were analysed using UV-visible spectrophotometer (PE $5400 \mathrm{UV}$ ) in the range of $190-800 \mathrm{~nm}$ using a quarts cell.

The friction and wear tests were performed by using tribometer in a pin-on-disc configuration (UMT-200, Converse Resource). The wear specimens (40X steel) in the form of pins with diameter $10 \mathrm{~mm}$ were slid against the $40 \mathrm{X}$ steel disc (diameter $80 \mathrm{~mm}$ ). Three pins were used during each test. Chemical composition of $40 \mathrm{X}$ steel is $\mathrm{C} 0.36 \%$, Si $0.17 \%$, Mn $0.5 \%$, Ni $0.3 \%$, S $0.035 \%$, P $0.035 \%$, Cr $0.8 \%$, Cu $0.3 \%$, Fe $\sim 98 \%$. The surface of the disc was processed uniformly by polishing. The testing was performed under normal loads $49 \mathrm{~N}, 98 \mathrm{~N}, 147 \mathrm{~N} 196 \mathrm{~N}, 245 \mathrm{~N}$ and at a rotating speed of $180 \mathrm{rpm}$ for duration 2 hours. 
The pins and the disc were cleaned with alcohol and acetone and dried prior to and after each test. Tribology test was conducted in room temperature and ambient atmosphere. Maximum temperature of lubricant around sliding contact was about $30{ }^{\circ} \mathrm{C}$. The glycerol was used as base liquid for tribology measurement. This polar solvent is suitable for creating stable dispersions of GO and allows to determine the effect of nanoparticles for wear of friction surfaces. GO was dispersed in glycerol by ultrasonication for 45 minutes. The lubricating compositions with 0.05 wt. \% GO and GO-CuO additives were obtained.

\section{Results and discussion}

The XRD pattern of GO is shown in Fig. 1a. The efficiency oxidation of graphite was estimated by shift of reflection assigned to the (002) plane. After oxidation, the diffraction peak at $2 \theta 26.6^{\circ}$ is moved to $13,5^{\circ}$. The formation of the hybrid nanomaterial "GO-CuO" is confirmed by XRD data. It can be seen, Fig. $1 \mathrm{~b}$ exhibits peaks corresponding to the monoclinic phase of copper (II) oxide. UV-vis absorption spectrum of GO (Fig. 1c) is characterized by maximum at $332 \mathrm{~nm}$ associated with $\pi-\pi^{*}$ transitions of C-C aromatic bonds and weak shoulder at $300 \mathrm{~nm}$ due to $\mathrm{n}-\pi^{*}$ transitions bonds $\mathrm{C}=\mathrm{O}$ [20].
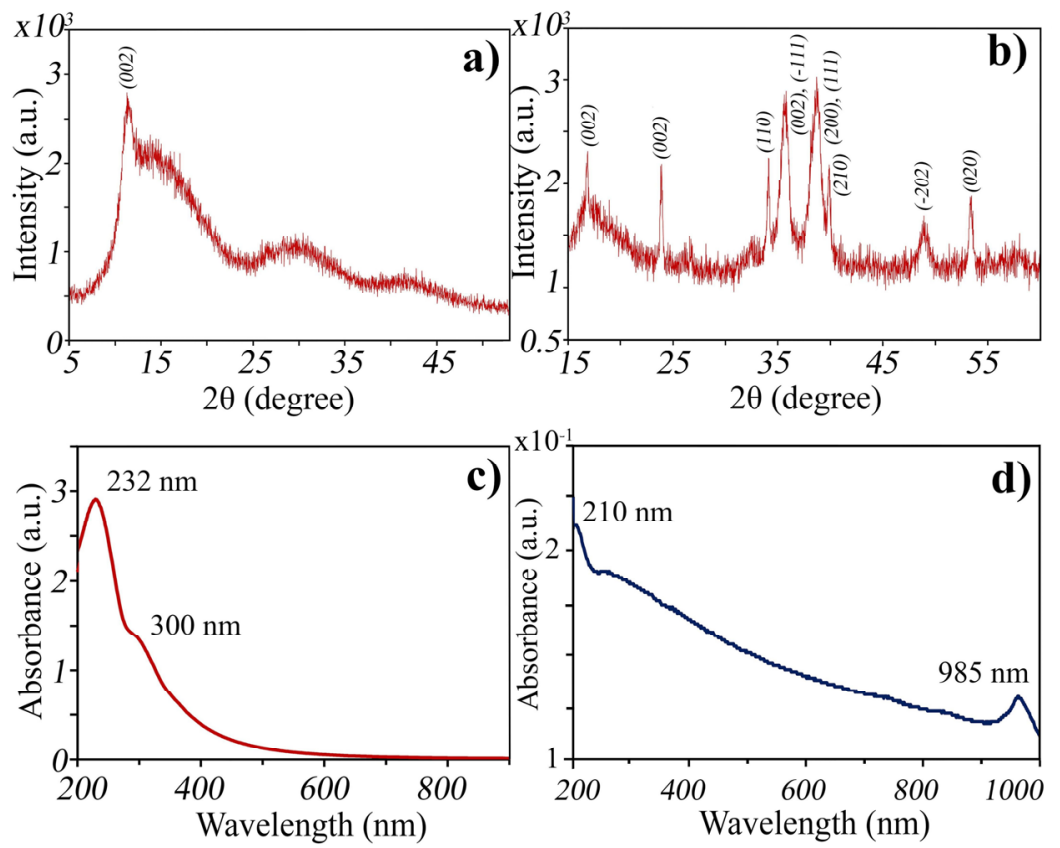

Fig. 1. XRD patterns of (a) GO and (b) GO-CuO. UV-vis spectra of GO (c) and GO-CuO (d).

Fig. 2 shows the dependence of the coefficient of friction on the time of tribological tests. It is obvious that the mechanisms of the lubricating action of graphene oxide and hybrid are different. A significant reduction in the friction coefficient in the dispersion of graphene oxide in comparison with the pure solvent is associated with the formation of a protective tribofilm on the surface of the rubbing contacts. It is worth noting that the coefficient of friction practically does not change with increasing load and is within the range of $\sim 0.02$. Lubricity of a hybrid material containing copper oxide nanoparticles is less pronounced. Under test conditions, the friction coefficient decreased from 0.05 at $49 \mathrm{~N}$ to $\sim 0.35$ at 196 and $245 \mathrm{~N}$. 


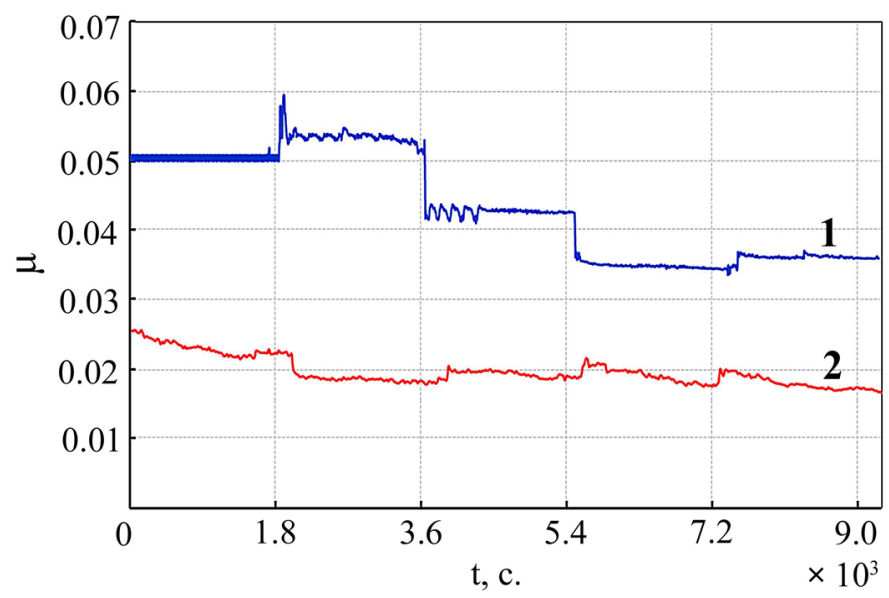

Fig. 2. The friction coefficient as a function of time of steel sample lubricated with GO (1) and $\mathrm{GO}-\mathrm{CuO}(2) 0.05$ wt. \% dispersions in glycerol.

The lubricating action of metal oxide nanoparticles can be associated with the formation of a third body that delimits frictional contacts. The formation of a protective film due to the melting of nanoparticles seems unlikely because of the high melting point of $\mathrm{CuO}$. Nevertheless, increasing the load leads to a decrease in the coefficient of friction. This suggests that under high pressure conditions a synergistic effect of the combined action of graphene oxide and $\mathrm{CuO}$ nanoparticles. In this connection, further studies of the lubricating effect of hybrid graphene materials, including the study of the surfaces of frictional contacts, are an actual scientific task.

\section{Conclusion}

Lubricant additives of graphene oxide and hybrid material containing $\mathrm{CuO}$ nanoparticles have a different effect on the friction coefficient in the tribosystem of steel-glycerol-steel. In the presence of graphene oxide, the coefficient of friction is reduced to $\sim 0.02$. The lubricating effect of graphene oxide is due to its layered structure, resulting in the formation of an extended tribofilm. When adding a hybrid material, the friction coefficient is reduced to $\sim 0.035$. Lubricating action of this material is associated with the formation of a third body in the frictional interaction zone.

The research was financially supported by the internal grant of the Don State Technical University (grant of L.V. Krasnichenko). The tribological researches on the tribometer were held in the Laboratory of Hybrid Functional Materials Based on Graphene of Research and Education Center "Materials" of Don State Technical University.

\section{References}

1. K.P. Loh, Q. Bao, P.K. Ang, J. Yang, J. Mater. Chem. 20(12), 2277 (2010)

2. Z. Sun, D.K. James, J.M. Tour, J. Phys. Chem. Lett., 2(19), 2425 (2011)

3. L.Y. Lin, D.E. Kim, W.K. Kim, S.C. Jun, Surf. Coat. Technol., 205(20), 4864 (2011)

4. D. Berman, A. Erdemir, A.V. Sumant, Mater. Today, 17(1), 31 (2014)

5. J.W. Jiang, J.S. Wang, B. Li, Phys. Rev. B: Condens. Matter, 80(11), 113405 (2009) 
6. D. Prasai, J.C. Tuberquia, R.R. Harl, G.K. Jennings, K.I. Bolotin, ACS nano, 6(2), 1102 (2012)

7. M.S. Cao, X.X. Wang, W.Q. Cao, J. Yuan, J. Mater. Chem. C, 3(26), 6589 (2015)

8. S. Liang, Z. Shen, M. Yi, L. Liu, X. Zhang, S. Ma, Carbon, 96, 1181 (2016)

9. H. Chen, T. Filleter, Nanotechnology, 26(13), 135702 (2015)

10. P. Kumar, M.F. Wani, Tribol. T., 61(2), 335 (2018)

11. B. Gupta, N. Kumar, K. Panda, V. Kanan, S. Joshi, I. Visoly-Fisher, Sci. Rep., 7, 45030 (2017)

12. O. Penkov, H.J. Kim, H. J. Kim, D.E. Kim, Int. J. Precis. Eng. Manuf., 15(3), 577 (2014)

13. B. Gupta, N. Kumar, K. Panda, S. Dash, A.K. Tyagi, Sci. Rep., 6, 18372 (2016)

14. J. Zhao, J. Mao, Y. Li, Y. He, J. Luo, Appl. Surf. Sci., 434(21) (2018)

15. D. Marchetto, P. Restuccia, A. Ballestrazzi, M.C. Righi, A. Rota, S. Valeri, Carbon, 116, 375 (2017)

16. V. Jaiswal, S. Umrao, R.B. Rastogi, R. Kumar, A. Srivastava, ACS Appl. Mater. Interfaces 8(18), 11698 (2016)

17. L. Zhang, Y. He, S. Feng, L. Zhang, Z. Jiao, Y. Zhan, Y. Wang, Ceram. Int., 42(5), 6178 (2016)

18. H. Li, L. Chen, Y. Zhang, X. Ji, S. Chen, H. Song et. al., Cryst. Res. Technol., 49(4), 204 (2014)

19. H.J. Song, X.H. Jia, N. Li, X.F. Yang, H. Tang, J. Mater. Chem., 22(3), 895, (2012)

20. L. Shahriary, A.A. Athawale, Int. J. Renew Energy Environ. Eng., 2(01), 58, (2014) 